\title{
A New Look on Biomineralization With X-ray Crystalline 3D Microscopy
}

\author{
$\underline{\text { Virginie Chamard }}^{1, *}$ \\ 1. Aix-Marseille Univ, CNRS, Centrale Marseille, Institut Fresnel, F-13013 Marseille, France \\ *virginie.chamard@fresnel.fr
}

Biomineralization, the capability exhibited by many living organisms to produce hard tissues (e.g., bones, teeth, shells) involves extraordinarily complex and regular biochemical processes whereby the organo-mineral components are controlled and organized with specific crystalline forms and textures $[1,2]$. Considering only calcium carbonate polymorphs in molluscs, corals or sponges, a variety of hierarchical sub-millimetric structures and shapes are observed [3]. Consequently, biominerals often present outstanding material properties, like increased damage resistance [4] or bone regeneration induction [5], motivating strong efforts for developing biomimetic materials. Furthermore, the incorporations of trace elements $(e . g ., \mathrm{Mg}, \mathrm{Sr}, \mathrm{Cd}, \mathrm{Ba})$ during the shell formation of $\mathrm{CaCO}_{3}$ marine species directly register the environmental parameters. Thus, these shells are used in paleoclimatology as proxies for ocean paleo-temperature, productivity and circulation [6]. To circumvent the lack of a general model for the trace element incorporation, extensive empirical laws are derived, for each species and environmental conditions [see e.g., 6]. The variations of trace element concentration, observed within the shells [7], further strengthen the need for a deep understanding of the processes underlying the formation of the biominerals. Finally, as $\mathrm{CaCO}_{3}$ is one of the most prominent minerals in the Earth's crust, it plays a major role in the global Carbon cycle [8]. Understanding the physico-chemical pathways underlying the synthesis of biomineral $\mathrm{CaCO}_{3}$ in marine species would pave the way for refined models of climate evolution [8].

Strategies to progress on the understanding of biomineralization involve ex vivo structural observations and in vitro synthesis [9-11]. Strong efforts are dedicated to the observation of the sub-micrometric scale: indeed, in contrast to the wide variety of architectures and crystalline polymorphs observed in biogenic shells, the sub-micrometric scale of biominerals evidences a granular organo-mineral interplay, whose systematic presence indicates a generic process for bio-crystallization [12]. The formation of the granular structure, characterized by rounded particles of $50-200 \mathrm{~nm}$ size, is not compatible with the classical crystallization theory, i.e., the successive addition of monomers onto a crystalline nucleus producing a well faceted single-crystal. Moreover, this granular sub-micrometric (mesoscale) structure shows the length scale at which to focus: one expects that the detailed characterization of the granular interplay would bring decisive evidences for deciphering the physico-chemical pathways at play during biomineralization. In a recent review article, De Yoreo et al. present a summary of different nonclassical crystallization physico-chemical pathways based on the concept of particles attachment. Each pathway is characterized by the initial involvement of different particles, from multi-ion complexes to droplet particles or single-nanocrystals [10]. In order to check the relevancy of one of these models for biomineralization, a detailed visualization of the 3D mesoscale crystalline structure is needed, for biogenic and biomimetic materials. However, none of the available microscopies (with electron, $\mathrm{x}$-ray or visible light) is able to fully answer this need.

In this context, we have proposed to use Bragg ptychography, an x-ray crystalline microscopy approach we developed in 2011 [13]. Bragg ptychography is a direct extension of ptychography [14], a lens-less 
microscopy method, which makes use of a series of coherent diffraction intensity patterns, obtained by scanning a finite size $\mathrm{x}$-ray probe across the sample. If the overlap between two successive positions is large, sufficient information redundancy is introduced in the data set. This redundancy is further exploited by inversion iterative algorithms, which phase back the intensity diffraction patterns and allow the retrieval of the sample complex-valued scattering function [15]. For crystalline microscopy, the measurement is performed in the vicinity of a Bragg reflection (figure 1a) and requires the introduction of a 3D approach for the measurement and the data inversion. The 3D intensity patterns are collected at each beam to sample position, by angularly scanning the sample along the rocking curve i.e., perpendicularly to the Bragg vector (figure 1b). In order to preserve the data integrity, the inversion is performed directly in the detection frame and the associated conjugated direct space, requiring expressing all quantities in a non-orthogonal frame [16]. After the inversion process, the 3D sample scattering function is transferred to the laboratory frame. It carries information on the crystalline displacement field, from which 3D strain, rotation and coherence length maps can be derived, with nanoscale resolution [17]. The relevancy of Bragg ptychography with respect to the biomineralization question is thereby evident.

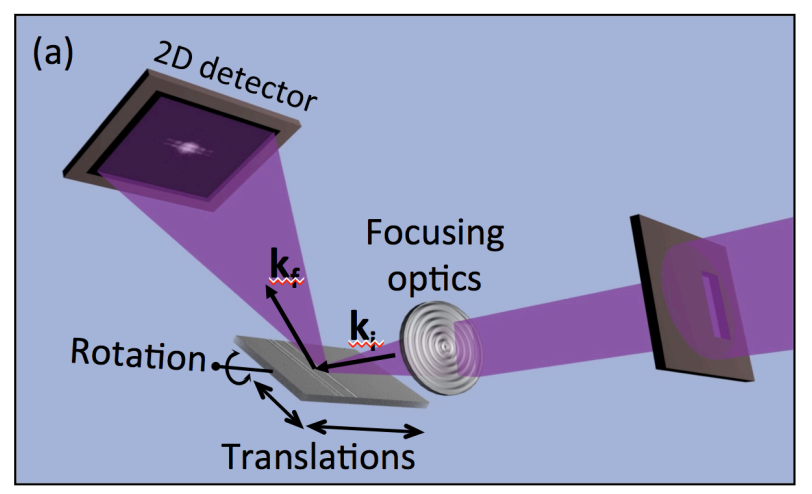

Figure. 1. (a) X-ray Bragg ptychography set-up including the focusing optics, the translation/rotation stage and the 2D far-field detector. (b) The 3D information is obtained by scanning angularly the sample in the vicinity of the Bragg peak. The incident and exit wave vectors $\mathbf{k}_{\mathrm{i}}$ and $\mathbf{k}_{\mathrm{f}}$ are indicated. Partially adapted from [13].

In a series of experiments performed at the ID13 beamline at ESRF [18], we have investigated the internal crystalline structure of a Pinctada margaritifera shell, a biomineral whose external layer is composed of single-crystalline prismatic units (figure 2(a-c)). The x-ray Bragg ptychography experiment was conducted in Laue geometry, in the vicinity of the 110 Bragg reflections (figure 2(d)), allowing us to image the 110 crystalline plane properties in a volume of about $1 \mu^{3}$ (figure 2(e)), with resolution of about $(30 \mathrm{~nm})^{3}$. The analysis of the 3D retrieved displacement field showed the coexistence of several iso-oriented and iso-strained domains, with dimensions in the order of a few granules (figure 2(f-i)). Each domain contains crystalline coherence domains, larger than the individual granules. These unique structural findings call for specific non-classical crystallization pathways, either involving partial fusion of oriented attached nanocrystal assembly [10] and/or the existence of liquid droplet precursors followed by a liquid to solid-amorphous and solid-amorphous to crystal phase transitions [11].

The wealth of information brought with this recently developed imaging method demonstrates that new findings on the understanding of the biomineralization process are within reach. The next steps will aim 
at investigating the crystalline structure in the vicinity of the crystallization growth front to discriminate between the two proposed models [19].
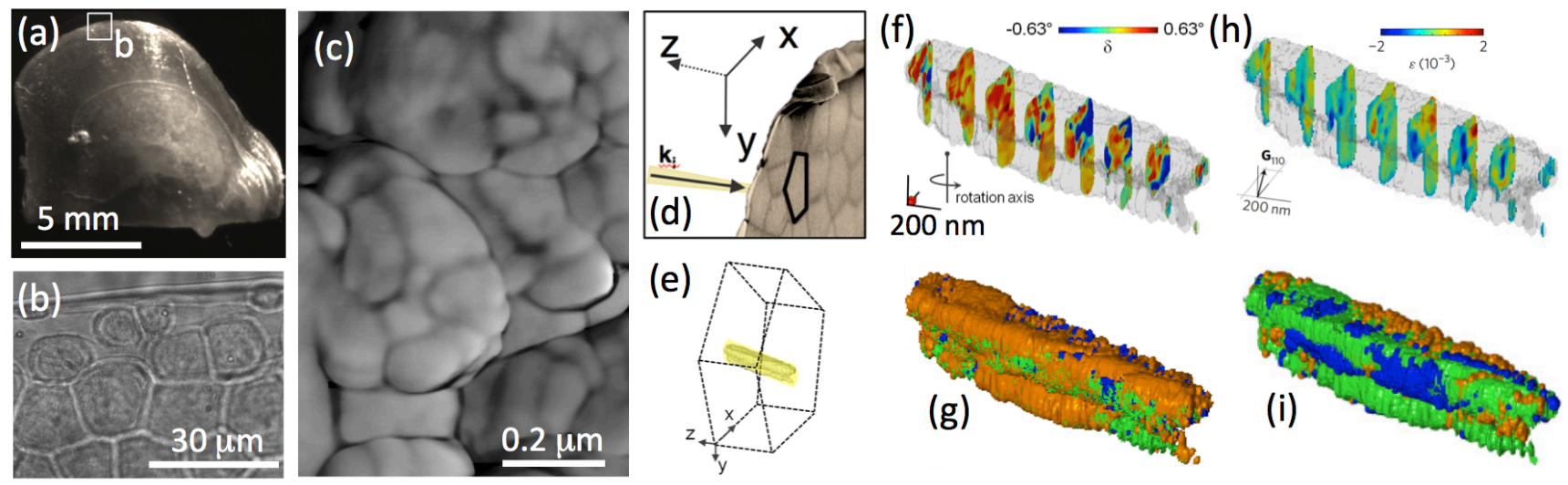

Figure. 2. (a) the Pinctada Margaritifera shell. (b) Zoom-in view of the shell border. (c) The granular mesoscale structure. (d) Experimental geometry and the investigated calcite prism. (e) The retrieved volume analysed from the x-ray Bragg ptychography data set. (f, g) Cross-sections of the crystalline lattice rotation $(\delta)$ maps and iso-surface rendering representation. (h, i) Same as $(f, g)$ for the strain $(\varepsilon)$ maps. Partially adapted from [18].

\section{References:}

[1] S. Weiner and L. Addadi, J. Mater. Chem. 7 (1997) 689.

[2] O. M. Boggild, Kgl Dan. Vidensk Selsk Skr. Natruvidensk Og Mathem 2 (1930) 231.

[3] J.-P. Cuif, Y. Dauphin and E. Sorauf, "Biominerals and fossils through time", Cambridge Univ.

Press (2011).

[4] L. Li and C. Ortiz, Adv. Funct. Mater 25 (2015) 3463.

[5] G. Atlan et al., C. R. Acad. Sci. 320 (1997) 253.

[6] D. Lea et al., Science 289 (2000) 1719.

[7] O. Branson et al., EPSL 383 (2013) 134.

[8] A. Ridgwell and R. Zeebe., EPSL 234 (2005) 199.

[9] F. Nudelman et al., Faraday Discuss. 136 (2007) 9.

[10] De Yoreo et al., Science 349 (2015) 6247.

[11] L. B. Gower, Chem. Rev. 108 (2008) 4551.

[12] Y. Dauphin, Mineral. Mag. 72 (2008) 243.

[13] P. Godard et al., Nat. Commun. 2 (2011) 568.

[14] J. M. Rodenburg and R. H. T. Bates, Phil. Trans. R. Soc. Lond. A 339 (1992) 521.

[15] M. Dierolf et al., Nature 467 (2010) 436.

[16] F. Berenguer et al., Phys. Rev. B 88 (2013) 144101.

[17] J. N. Clark et al., Nat. Mater. 14 (2015) 780.

[18] F. Mastropietreo et al., Nat. Mater. 16 (2017) 946.

[19] The author acknowledges funding from the French ANR (ANR-11-BS20-0005) and the European Research Council (ERC) for the European Union's Horizon H2020 research and innovation programme grant agreement No 724881. ESRF is acknowledged for providing access to the source and beamlines. This article is largely based on Ref. [18], whose authors are warmly acknowledged. The author whishes to thank S.O. Hruszkewycz and A. Talneau, who have accompanied us in this field over the past years. 
https://doi.org/10.1017/S1431927618012461 Published online by Cambridge University Press 